\title{
Tsafon
}

Revue d'études juives du Nord

$74 \mid 2017$

La Déclaration Balfour, vers l'État d'Israël?

\section{« La vieillesse ne signifie pas la fin, mais la moisson »}

Andrée Lerousseau

\section{(2) OpenEdition}

1 Journals

Édition électronique

URL : https://journals.openedition.org/tsafon/409

DOI : $10.4000 /$ tsafon.409

ISSN : 2609-6420

Éditeur

Association Jean-Marie Delmaire

Édition imprimée

Date de publication : 1 décembre 2017

Pagination : 133-142

ISSN : 1149-6630

\section{Référence électronique}

Andrée Lerousseau, « «La vieillesse ne signifie pas la fin, mais la moisson » », Tsafon [En ligne], 74 |

2017, mis en ligne le 31 mai 2018, consulté le 25 juin 2021. URL : http://journals.openedition.org/ tsafon/409; DOl : https://doi.org/10.4000/tsafon.409

Tsafon. Revues d'études juives du Nord 
Varia : Nelly Sachs

\section{« La vieillesse ne signifie pas la fin, mais la moisson $\iota^{1}$}

\section{Andrée Lerousseau*}

Située à proximité du parc s'étendant au nord de la ville, la maison de retraite de la communauté juive de Düsseldorf, le Nelly-Sachs-Haus (la Maison Nelly Sachs), fut conçue à l'origine pour accueillir les parents des membres de la communauté ayant effectué un retour en Allemagne. Elle se définit d'ailleurs comme un «foyer destiné aux parents » (Elternheim), en référence, ainsi que le souligne Bert Römgens, directeur de l'établissement, aux deux piliers du judaïsme que sont les enfants et les parents ${ }^{2}$. La capacité d'accueil a été accrue lors de la restructuration et de la rénovation à la fin des années quatre-vingt-dix, passant de 80 places à l'origine à 110. Dans ce microcosme se côtoient des Juifs allemands, d'autres venus d'Israël, et un grand nombre de pensionnaires originaires des territoires de l'ancienne Union Soviétique (Ukraine,

\footnotetext{
* Université de Lille-SHS. Je tiens ici à remercier Madame Annette Brandt, pour son chaleureux accueil au Nelly-Sachs-Haus en mars dernier, et Madame Theresia Schüllner qui avait pris le soin d'organiser cette rencontre. L'équipe de Tsafon exprime sa reconnaissance à Monsieur Bert Römgens, directeur du Nelly-Sachs-Haus, pour son autorisation de publier cet article.

${ }^{1}$ «Das Alter ist nicht das Ende, sondern die Ernte »: telle est la devise, empruntée à Ernst Bloch, de la Maison Nelly Sachs à Düsseldorf.

${ }^{2}$ Voir l'article de Larissa Lee Beck paru en janvier 2015 dans l'édition régionale de Die Welt, " Der Lebensabend in einem jüdischen Seniorenheim », https://www.welt.de > Regionales > Nordrhein-Westfalen.
} 
Russie, Ouzbékistan), pour la plupart bien intégrés en Allemagne depuis des années et ayant une maîtrise parfaite de la langue, à l'instar de Betti Khaikina, octogénaire et ancienne directrice d'un service de neurologie à Moscou, qui confiait à Frank Rothert qui rédigea en 2008 un article pour la Jüdische Allgemeine, le journal de la communauté juive de Düsseldorf, que si son âme était certes demeurée à Moscou, elle se sentait ici comme chez elle $^{3}$. Il convient également de souligner que l'établissement héberge, sur leur demande, des catholiques, des protestants, des libres penseurs ou athées, et des membres des églises chrétiennes dites libres (Freikirchler).

Si la population de la résidence a ainsi suivi une évolution et une mutation analogues à celles de la communauté juive de la ville rassemblant aujourd'hui quelques 7000 membres dont une grande majorité sont issus de l'ancien bloc soviétique ${ }^{4}$, et si l'on dénombre $60 \%$ de russophones contre $40 \%$ de germanophones parmi les pensionnaires, le personnel étant en règle générale bilingue, Bert Römgens tient toutefois à préciser que le Nelly-Sachs-Haus n'est «pas une institution russe, mais une institution juive où l'on parle également le russe $»^{5}$. D'où le soin particulier porté au maintien de la tradition et de la culture juives, la célébration du shabbat et des fêtes juives au sein même de la maison de retraite, sous le plafond orné de l'étoile de David de la grande salle faisant office de synagogue et renfermant une bibliothèque où se réunit le cercle de lecture, des audiences avec le rabbin, le respect de la nourriture cachère, etc. Ainsi qu'il est stipulé dans le dépliant de présentation du Nelly-Sachs-Haus, on part en effet du principe qu'une «vie dans la dignité » signifie nécessairement « une participation active à la vie juive », un principe que n'aurait certes pas renié celle qui, peu avant sa mort en mai 1970 à Stockholm où elle résidait depuis 1940, accepta de donner son nom à la maison de retraite et qui partagea avec Agnon en 1966 le Prix Nobel de littérature « pour sa remarquable œuvre lyrique et dramatique qui interprète le destin d'Israël avec sensibilité et force ». À

\footnotetext{
${ }^{3}$ Frank Rothert, «Wie im richtigen Leben », article paru en août 2008 dans la Jüdische Allgemeine, www.juedische-allgemeine.de/article/view/id/2240. L'auteur rapporte les paroles de Betti Khaikina: "Meine Seele ist in Moskau, dennoch fühle ich mich zu Hause ».

${ }^{4}$ Pour l'historique de la communauté juive de Düsseldorf qui compta le poète Heinrich Heine parmi ses enfants (la première synagogue fut d'ailleurs édifiée en 1712 à l'instigation d'un ancêtre de Heine), voir le site https://www.jgd.de/

${ }^{5}$ Cité dans Frank Rothert, «Wie im richtigen Leben », op. cit. : « [...] wir sind primär keine russische, sondern eine jüdische Einrichtung, wo auch russisch gesprochen wird ».
} 
l'occasion du soixantième anniversaire de la remise du prix à celle que le poète Hans Magnus Enzensberger qualifiait de « dernière poétesse de la judaïté de langue allemande $\aleph^{6}$, une lecture de ses poèmes fut d'ailleurs organisée à l'attention des pensionnaires de la maison de retraite.

On est frappé, lorsque l'on pénètre dans le hall et la cafétéria de l'établissement, par le relatif bien-être qui se dégage du lieu et par ce que nous qualifierions, à défaut d'un autre terme peut-être plus approprié, de « normalité ». Tout est " comme dans la vraie vie », ainsi que le souligne le titre de l'article déjà cité de Frank Rothert. À moins, préfèrerions-nous dire, que ce ne soit tout simplement la vie qui se poursuit, dans une ambiance conviviale et un cadre agréable où l'on peut converser ou recevoir parents et amis dans le petit salon Nelly Sachs attenant à la cafétéria ou sur l'une des terrasses, flâner ou, à défaut d'autonomie, demeurer assis dans le «jardin des sens » (Sinnesgarten) où résonnent doucement, parmi les plantes et les fleurs naturelles, des fleurs de laiton, et se retrouver dans la salle à manger aux couleurs d'Israël.

La vie suit son cours avec bien sûr les petits accrochages, des mots échangés pouvant donner lieu à de longues controverses. Ainsi les « quelques remous» provoqués parmi les résidents non originaires de l'Europe de l'Est lorsque le bortsch figura pour la première fois au menu. Ou cette inscription anonyme gravée sur une table du jardin : «Je ne supporte plus d'entendre la langue russe », une langue renvoyant certains retraités venus de Roumanie à l'idiome des anciens occupants. Et on pourrait également évoquer ce reproche fait par un «Jecke » d'Israël à un Juif de Russie qu'il accusait de ne pas être « un vrai Juif», car il ajoutait du beurre à sa viande. Mais qu'est-ce qu'un «vrai Juif»? Voici bien de quoi susciter immédiatement le débat. Quant à l'humour, il n'est bien sûr pas absent, comme lorsque Tilde Schottenfeld, nonagénaire née à Vienne qui a grandi à Czernowitz et n'a pas eu moins de cinq nationalités dans sa vie, se qualifie, afin de souligner la longue et lourde expérience qui fut la sienne et de mettre en exergue peut-être sa conscience de soi en tant que femme, de « Mathusalem féminine » («weiblicher Methusalem ») ${ }^{7}$.

Si le souvenir de la Shoah et des persécutions demeure vivant, voire omniprésent, le sujet est toutefois rarement abordé. «Quand une

\footnotetext{
${ }^{6}$ « Nelly Sachs ist die letzte Dichterin des Judentums in deutscher Sprache », cité dans Thomas Sparr, notice consacrée à Nelly Sachs dans le Metzler Lexikon der deutschjüdischen Literatur, herausgegeben von Andreas B. Kilcher, Stuttgart-Weimar, Verlag J. B. Metzler, 2000, p. 503.

${ }^{7}$ Sur ces anecdotes, voir Frank Rothert, « Wie im richtigen Leben », op. cit..
} 
mère dont le fils a été assassiné à Auschwitz vit ici, comment venir à bout de cela», demande Bert Römgens, avant d'ajouter: «C'est impossible. On peut simplement être là pour l'autre et tenter de comprendre sa douleur $»^{8}$. Si Edith Bader-Devries accepte d'en parler, elle qui s'est tue si longtemps, c'est parce que la vieille dame entend aujourd'hui faire entendre ceux que l'on a réduits au silence'. Pour la journaliste qui l'interroge, elle extrait d'un classeur une image en couleur représentant une enfant qui tient une poupée dans ses bras, avec en arrière-plan le camp de Theresienstadt (une ancienne image de propagande?). Et elle raconte sa déportation dans ce camp avec ses parents en 1942, les souffrances de son père atteint par trois fois du typhus suite à la malnutrition et les nuits passées au milieu des morts, sans même une poupée pour se consoler, car on lui avait volé son enfance. Derrière le silence de la plupart des pensionnaires, le traumatisme demeure, explique Bert Römgens, et durant les grandes manifestations contre Israël de l'été 2014 et face à la montée de Pegida ${ }^{10}$, « la peur était palpable » ${ }^{11}$. L'établissement est d'ailleurs placé sous protection policière. Mise en place au lendemain des attentats du 11 septembre 2001, la surveillance a été renforcée après l'assassinat des journalistes de Charlie Heddo et la prise d'otages du magasin Hyper Cacher ayant entraîné la mort de trois clients et d'un employé, en janvier 2015 à Paris.

L'ombre du passé et les inquiétudes face à l'actualité n'occultent nullement l'attention portée au présent et à ses mutations et à l'avenir.

\footnotetext{
${ }^{8}$ Cité dans Larissa Lee Beck, « Der Lebensabend in einem jüdischen Seniorenheim », op. cit. : " "Wie arbeitet man auf, wenn eine Mutter hier lebt, deren Sohn in Auschwitz ermordet wurde", fragt Römgens. "Das kann man nicht. Man kann nur für den anderen da sein, versuchen sein Leid zu verstehen" ».

${ }^{9}$ Ibid. : « Jetzt rede ich für die Verstummten ».

${ }^{10}$ Pegida (Patriotische Europäer gegen die Islamisierung des Abendlandes / Patriotes Européens contre l'Islamisation de l'Occident) est un mouvement populiste qui a vu le jour à Dresde en octobre 2014. Concernant les manifestations pro-palestiniennes, accompagnées de paroles et de dérapages antisémites, qui se déroulèrent durant l'été 2014, nous tenons ici à rappeler l'admirable discours tenu à Berlin le 14 septembre 2014 par Angela Merkel devant le Conseil Central des Juifs d'Allemagne (Zentralrat der Juden in Deutschland) : « [...] nous affirmons clairement et sans équivoque que la vie juive nous appartient. Elle est partie intégrante de notre identité et de notre culture. [...] Nous devinons parfaitement l'intention perfide de l'antisémitisme qui est de faire des Juifs des marginaux dans notre pays. Contre cela, nous professons clairement : nos amis, voisins et collègues juifs - tous sont ici, en Allemagne, chez eux. » (notre traduction). Pour l'intégralité du discours, voir le site https://www.bundesregierung.de/.../Rede/2014/.../2014-09-14-mer...

${ }^{11}$ Larissa Lee-Beck, « Der Lebensabend in einem jüdischen Seniorenheim », op. cit..
} 
L'évolution tendant à privilégier le maintien et les soins à domicile et à n'avoir recours aux structures collectives qu'en cas de perte d'autonomie, la maison de retraite a développé tout un programme en direction des personnes âgées nécessitant un suivi médical lourd ou atteintes de démence, avec la création, pour ces dernières, d'un groupe d'activité s'attachant à maintenir d'une part le sens de la perception grâce à l'ergothérapie, par la stimulation des impressions et des sensations tactiles, olfactives, visuelles et acoustiques et au contact des objets familiers, et d'autre part la capacité à communiquer par la définition, entre autres, de rituels ${ }^{12}$. Dans un tout autre domaine, l'un des derniers projets en cours, en partenariat avec la résidence pour seniors Rishonei HaCarmel de Haïfa, vise à jeter un pont et à instaurer un dialogue, par le biais des réseaux et des outils de communication virtuelle, entre les pensionnaires des deux institutions, et à encourager les échanges et la collaboration au sein du personnel, dans le cadre entre autres du service volontaire israélo-allemand (Deutsch-Israelischer Freiwilligendienst) ${ }^{13}$.

Nous évoquerons pour conclure deux célèbres pensionnaires du Nelly-Sachs-Haus dont l'esprit et l'engagement ne nous semblent pas étrangers à ceux des fondateurs et directeurs de la maison de retraite. Née en 1921 à Berlin, Lili Marx est décédée en avril 2004 à Düsseldorf. Emigrée en Angleterre en 1939 (ses parents furent exterminés par les nazis), elle rentra en 1946 en Allemagne où elle participa activement, et non sans rencontrer des difficultés, à la reconstruction de la vie juive. Son premier mari, Karl Marx ${ }^{14}$, fonda la Jüdische Allgemeine, à l'époque le seul hebdomadaire juif dépassant le simple cadre régional en Allemagne, auquel elle collabora. De son côté, Lili Marx créa en 1949 le Jüdischer Frauenverein (Association des Femmes Juives) de Düsseldorf, avant de remettre sur pied en 1953, en compagnie de Jeanette Wolff, de Ruth Galinski et de Inge Marcus, le Jüdischer Frauenbund (JBF, Alliance des Femmes Juives) interdit en 1938. En 1951, elle et son mari

\footnotetext{
${ }^{12}$ Cf. à ce propos l'article d'Annette Kanis, publié en juin 2010 dans la Jüdische Allgemeine, à l'occasion des 40 ans de la résidence : "Alt werden leicht gemacht », http://www..juedische-allgemeine.de/articke/view/id/7869

${ }^{13}$ Le projet s'intitule «Altenpflegebrücke » (une passerelle dans les soins réservés aux personnes âgées). Voir à ce sujet https://www.duesseldorf.de/.../oberbuergermeisterthomas-geisel-b...

${ }^{14}$ Elle épousa par la suite l'écrivain israélien Alexander Czerski, partagea son temps entre Israël et l'Allemagne où elle rentra définitivement en 1986, et participa aux activités de la WIZO (Women's International Zionist Organisation).
} 
s'engagèrent enfin en faveur du dialogue et de l'amitié entre juifs et chrétiens ${ }^{15}$.

Sœur en poésie de Nelly Sachs, Rose Ausländer, née en 1901 à Czernowitz, s'installa à la fin des années soixante, à l'issue d'une vie d'exil et d'errance, dans une pension à Düsseldorf et résida à partir de 1972, suite à un accident qui lui fit perdre toute autonomie, dans la maison de retraite médicalisée, où elle mourut au début de janvier 1988. C'est là qu'elle rédigea nombre de recueils, dont le dernier, écrit en quelques nuits, intitulé Je compte les étoiles de mes mots. On perçoit dans l'un de ses poèmes les doux effluves du «jardin des sens» sur lequel donne aujourd'hui la fenêtre du petit salon qui porte son nom :

Cette fleur

Est belle à l'oeil

\author{
J'ignore \\ son nom \\ mais non son parfum \\ qui me revivifie ${ }^{16}$
}

Et peut-être son chant berce-t-il les conversations des pensionnaires dans la cafétéria toute proche :

\author{
La harpe \\ est mon instrument \\ Je joue \\ le chant de la vie ${ }^{17}$
}

\footnotetext{
${ }^{15}$ Ils furent co-fondateurs de la Gesellschaft für christlich-jüdische Zusammenarbeit (Société pour la coopération entre chrétiens et juifs) de Düsseldorf. Pour un excellent résumé de la vie et de l'itinéraire de Lili Marx, voir le site https://www.betdebora.net/de/lesestoff/lilli-marx-2/

${ }^{16}$ Rose Ausländer, Je compte les étoiles de mes mots, éd. bilingue, poèmes traduits de l'allemand par Edmond Verroul, Lausanne, éd. L'Âge d'Homme, 2000, p. 57 : « Diese Blume/ ist augenschön // ich weiß nicht/ ihren Namen/ nur den Duft/ der mich erquickt ».

${ }^{17}$ Ibid., p. 59 : « Die Harfe/ ist mein Instrument // Ich spiele/ das Lebenslied».
} 


\section{Nelly-Sachs-Haus (Düsseldorf)}

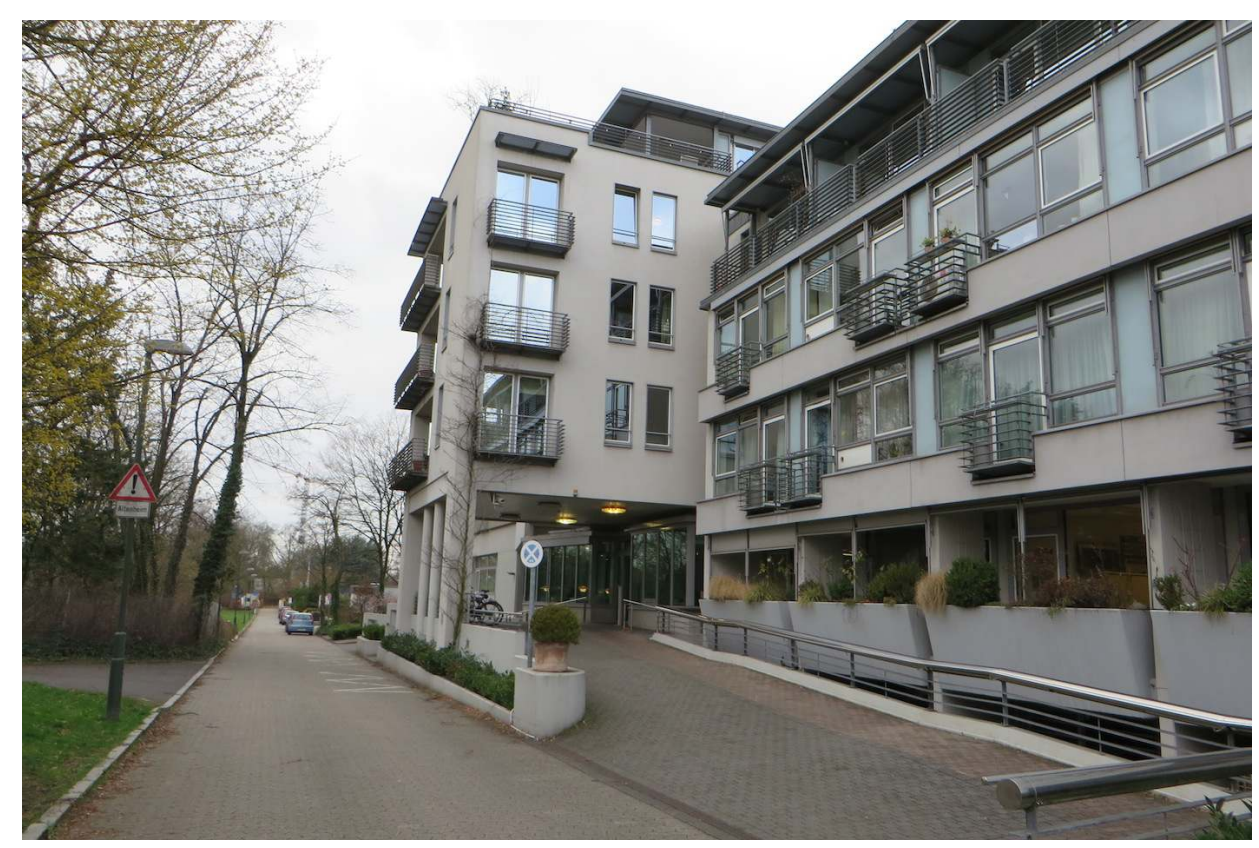

Vue extérieure

(Photo : Andrée Lerousseau) 
Nelly-Sachs-Haus (Düsseldorf)

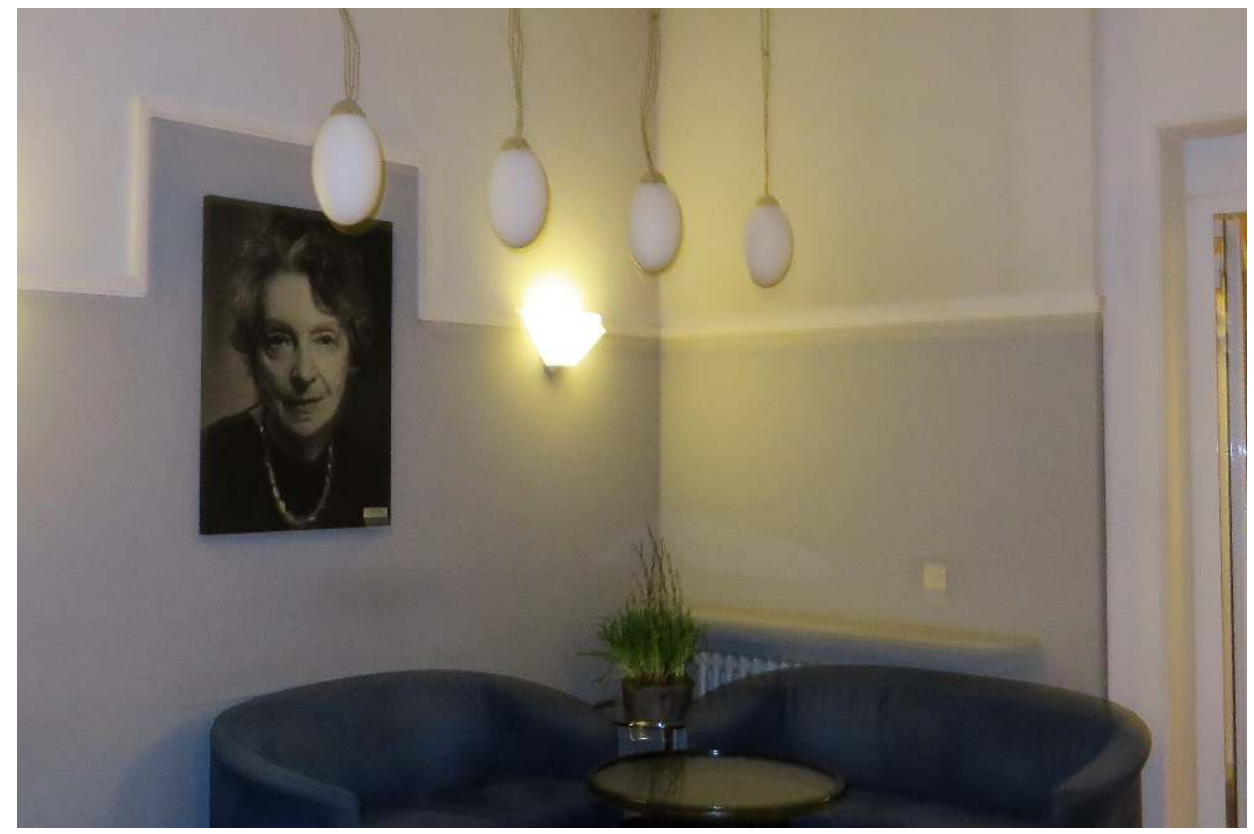

Salon de Nelly Sachs

(Photo : Andrée Lerousseau) 
Nelly-Sachs-Haus (Düsseldorf)

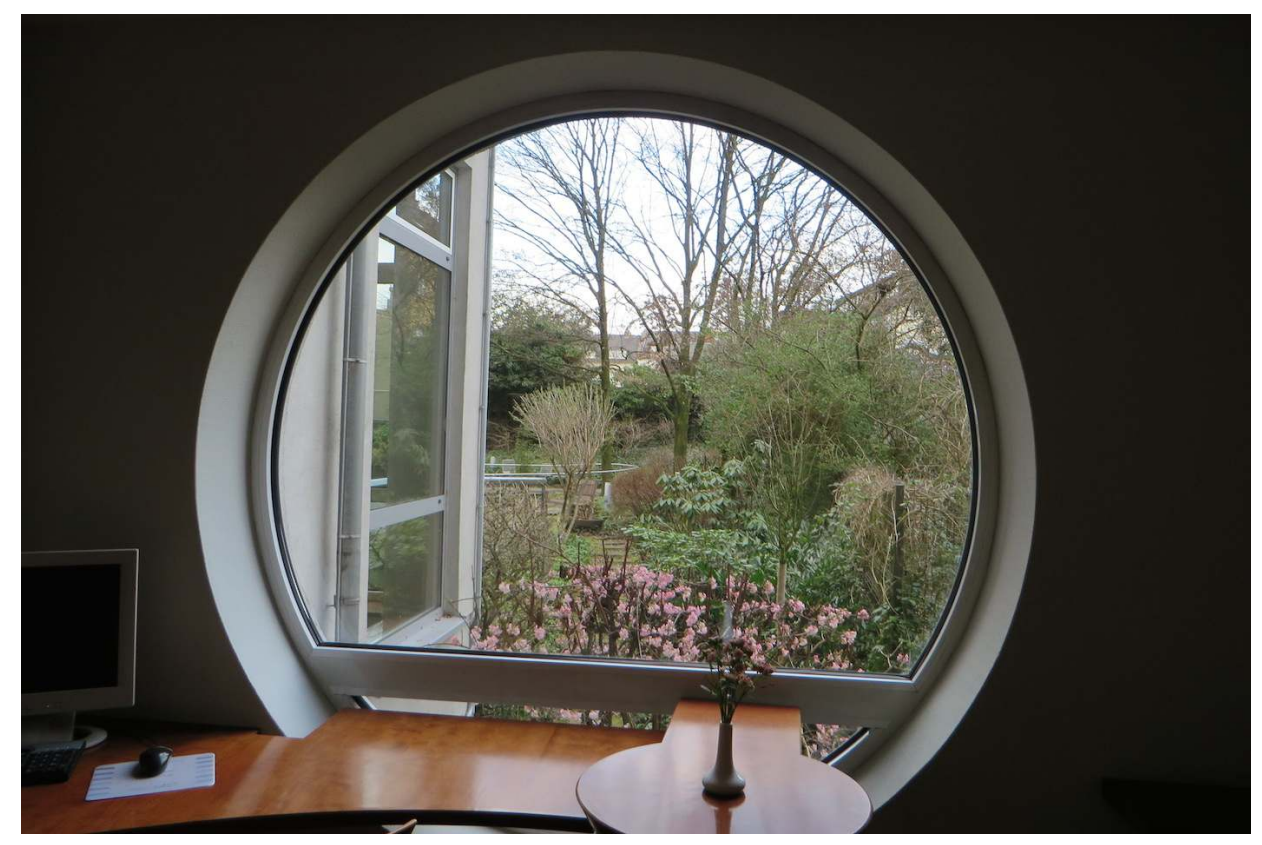

Le jardin des sens

(Photo : Andrée Lerousseau) 
Nelly-Sachs-Haus (Düsseldorf)

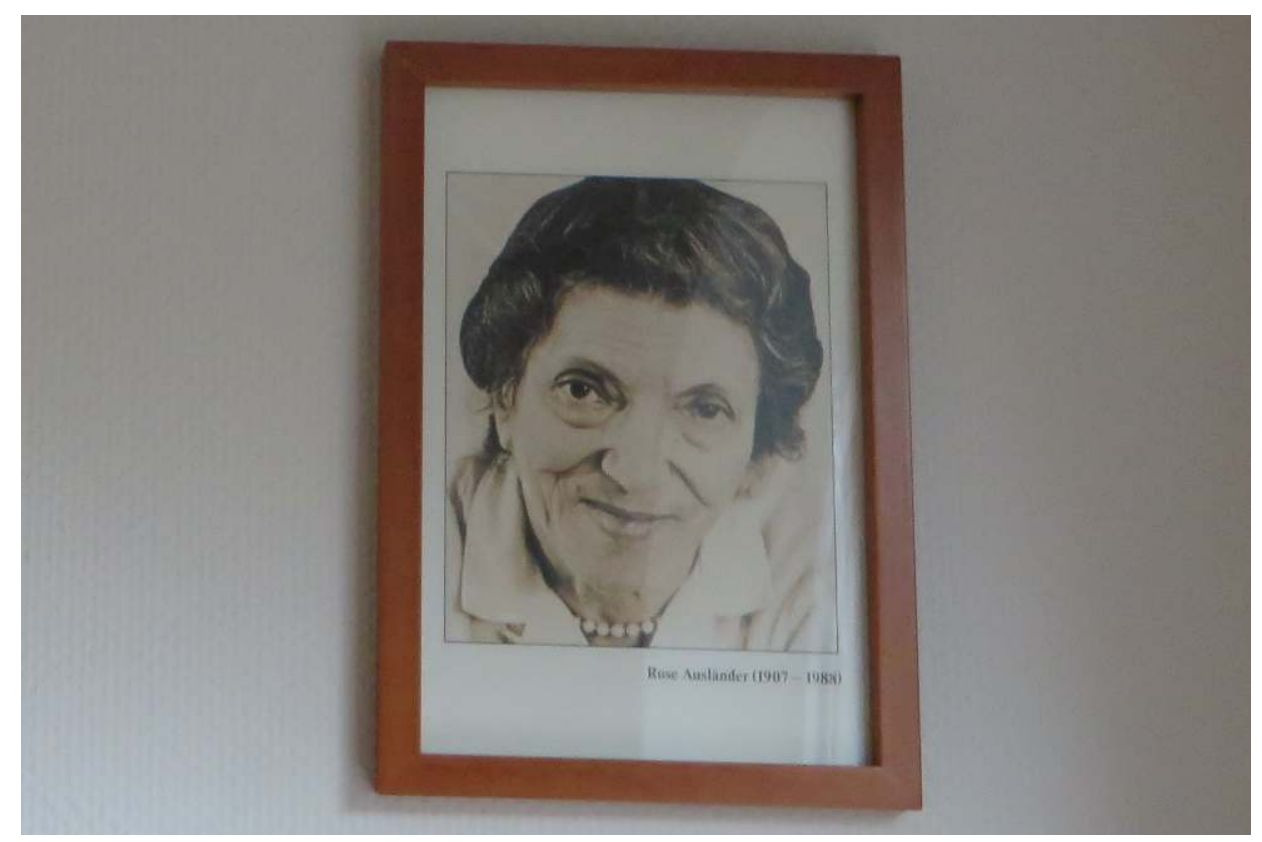

Le petit salon Rose Ausländer

(Photo : Andrée Lerousseau) 